\title{
Advanced Characterization of Material Properties on the Nanometer Scale Using Atomic Force Microscopy
}

\author{
M.A. Fenner ${ }^{a, *}$, S. Wu ${ }^{b}$, J.-J. Yu ${ }^{b}$, H.-P. Huber ${ }^{c}$ And F. Kienberger ${ }^{a}$ \\ ${ }^{a}$ Agilent Technologies GmbH, Kronberg, Germany \\ ${ }^{b}$ Agilent Technologies Inc., Chandler, USA \\ ${ }^{c}$ Christian-Doppler-Laboratory, Johannes Kepler University, Linz, Austria
}

\begin{abstract}
We report recent advances in material characterization on the nanometer scale using scanning microwave microscopy. This combines atomic force microscopy and a vector network analyzer using microwave tip sample interaction to characterize dielectric and electronic material properties on the nanometer scale. We present the methods for calibration as well as applications. Scanning microwave microscopy features calibrated measurements of: (1) capacitance with attofarad sensitivity. For calibration a well characterized array of capacitors $(0.1 \mathrm{fF}$ to $10 \mathrm{fF}$ ) is used. The method is applied to determine the dielectric properties of thin organic films, (2) Semiconductor dopant density. Calibration is performed by imaging the cross-section of a standard sample with differently doped layers (dopant stair case) from $10^{16}$ atoms $/ \mathrm{cm}^{3}$ to $10^{20}$ atoms $/ \mathrm{cm}^{3}$.
\end{abstract}

PACS: 77.55.-g, 07.79.-v, 68.37.Ps, 68.37.Uv, 07.57.Pt

\section{Introduction}

Since the introduction of atomic force microscopy (AFM) by Binnig et al. [1] it has been widely accepted as a powerful tool for high resolution imaging of surfaces on the nanometer scale and nanometer sized objects attached to surfaces. Beyond high resolution imaging the AFM has been used to measure material properties with highest resolutions. An overview can be found e.g. in $[2,3]$. Electrical and electronic properties have been studied with a variety of techniques. These include - among others - scanning microwave microscopy (SMM).

SMM combines AFM with microwave signal analysis to measure a variety of sample properties including complex dielectric constant [4-9], sheet resistance [1, 10] and tipsample capacitance [11-13]. SMM has also been shown to be sensitive to magnetic domain structure [14] and photovoltaic response $[15,16]$. Near field microscopy at microwave frequencies has also been applied via tapping mode technique [17].

\section{Scanning microwave microscopy 2.1. Implementation}

The SMM presented here connects a standard beam deflection AFM (Agilent 5420 or Agilent 5600) to a microwave vector network analyzer (e.g. Agilent PNA) via

\footnotetext{
* corresponding author; e-mail: matthias_fenner@agilent.com
}

a microwave (MW) coaxial cable. The PNA sends a continuous MW signal to the conducting AFM tip and detects the reflected MW signal. The PNA acts as a reflectometer and the normalized reflection signal is called $S_{11}$. The amount of reflected signal depends on the load impedance. For a standard AFM tip sample junction it is mainly capacitive in the $\mathrm{fF}$ range presenting several $\mathrm{k} \Omega$ of impedance.

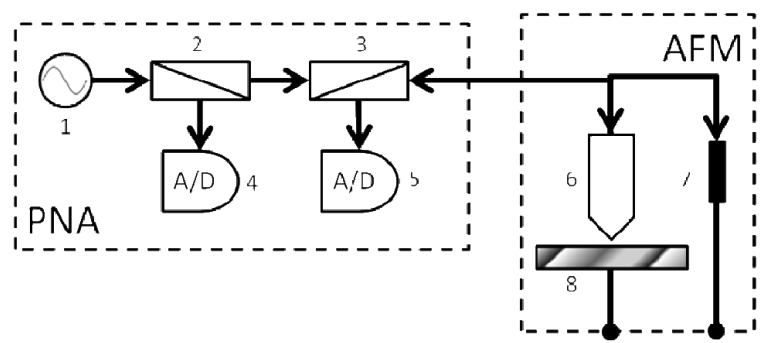

Fig. 1. Schematic setup of SMM: 1 - microwave source, 2 - directional coupler for power reference, 3 - directional coupler for reflected signal power, 4 data acquisition for power reference, 5 - data acquisition for reflected signal power, 6 - AFM scanner and tip, $7-50 \Omega$ load in resonator setup with AFM tip, 8 - sample.

Figure 1 shows a simplified block diagram of a PNA reflectometer setup. The modern PNAs are based on a $50 \Omega$ characteristic impedance, thus measurement accuracy 
and resolution suffer markedly when load impedances substantially deviate from $50 \Omega$. The use of a $50 \Omega$ shunt in conjunction with a half-wavelength coaxial resonator can solve this measurement problem. The implementation of a resonator implies that the SMM must be operated at a set of frequencies at which the MW half wavelength matches the resonator length. In the setup presented here frequencies from 1 to $18 \mathrm{GHz}$ can be used. When the AFM tip is scanned across the sample surface the reflected signal varies according to the varying tip sample capacitance.

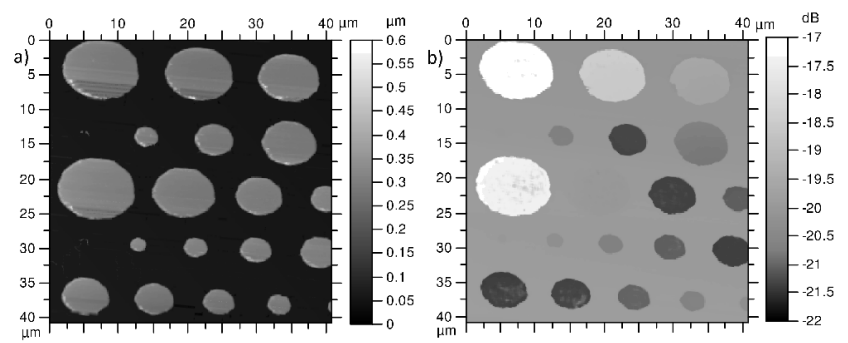

Fig. 2. SMM data of an array of gold islands of different sizes resulting in different capacitances. (a) AFM contact mode topography and (b) the corresponding map of the reflected MW signal. PNA parameters were set to measure the signal relative to the central gold pad, thus larger gold pads exhibit higher $S_{11}$ values, and vice versa.

The reflected signal is recorded simultaneously to the topography as illustrated in Fig. 2. An array of gold pads of different capacitances and the corresponding map of the reflected signal is imaged. In this case the PNA parameters were set to measure the signal relative to the central gold pad, thus larger gold pads exhibit higher $S_{11}$ values, and vice versa.

\subsection{Calibration}

Modern PNAs return a calibrated impedance value for standard experimental configurations but the use of a resonator setup requires additional calibration. This is

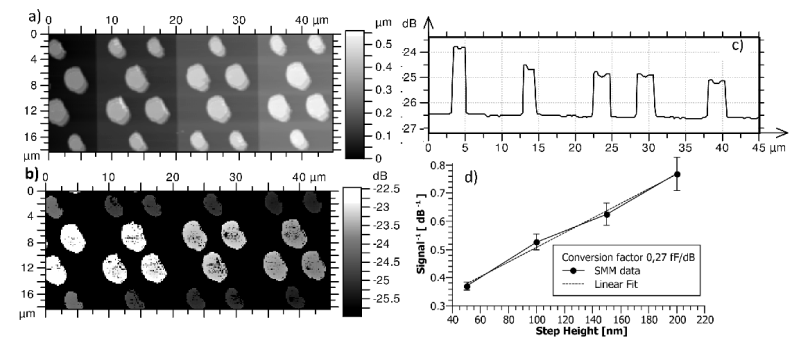

Fig. 3. SMM data of a capacitance calibration standard sample. (a) AFM contact mode topography showing increasing step height from left to right, (b) the corresponding map of the reflected MW signal $S_{11}$, (c) cross-section through the $S_{11}$ signal along the set of capacitors with $2 \mu \mathrm{m}$ diameter, (d) plot of $1 / S_{11}$ versus dielectric thickness. The linear fit converts to a conversion factor $0.27 \mathrm{fF} / \mathrm{dB}$. performed by scanning the tip over a well characterized array of capacitors. The resulting map of reflected signal can then be used to generate a transfer function relating the reflected signal to the tip-sample capacitance.

Figure 3 shows topography and reflected signal of a calibration standard provided by NIST [18]. The capacitors top electrodes are gold islands grown on a staircase of silicon oxide dielectric and the highly doped silicon wafer acts as a back electrode. The diameters range from 1 to $4 \mu \mathrm{m}$ and thicknesses from 50 to $200 \mathrm{~nm}$ effectively forming plane plate capacitors. It has been shown that a stray capacitance in the dielectric to semiconductor interface can be eliminated by plotting the inverse signal $\left(S_{11}\right)^{-1}$ versus the dielectric thickness and fitting the slope [18]. The resultant transfer function is a simple coefficient, see Fig. 3.

\subsection{Application to thin films}

SMM has been used to characterize a mixed self assembled monolayer (SAM) of octadecanethiol and decanethiol (referred to as $\mathrm{C} 18$ and $\mathrm{C} 10$, respectively) on gold, Fig. 4. A $2 \mu \mathrm{m} \times 2 \mu \mathrm{m}$ square of $\mathrm{C} 18$ was fabricated into the extended C10 SAM by nanografting [19]. The molecular conformation and packing structures of $n$ -alkanethiol molecules within a SAM have been well characterized $[20,21]$. The layer thickness of the C10 SAM is $1.32 \mathrm{~nm}$ while that of the C18 SAM is about $2.2 \mathrm{~nm}$, leading to a variation of $0.88 \mathrm{~nm}$ in height between the two different assemblies. This cannot be distinguished on the much rougher gold substrate with a corrugation of several tens of nanometers, Fig. 5 .

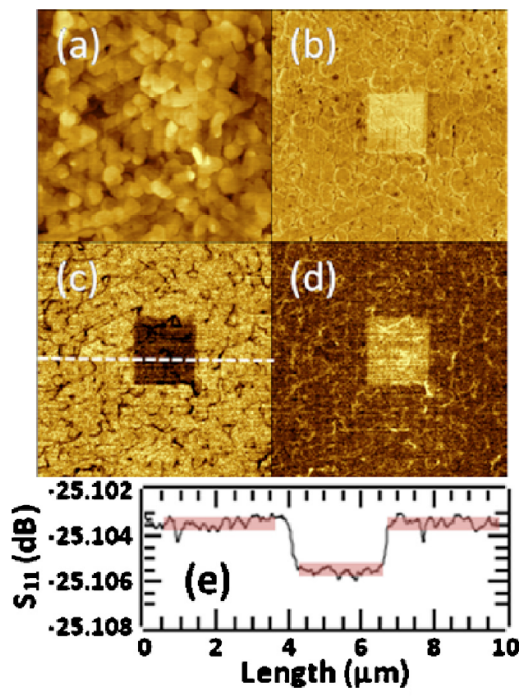

Fig. 4. SMM imaging of a C18/C10 SAM structure on $\mathrm{Au}$ (111) surface: (a) topography, (b) friction, (c) S11 amplitude, (d) S11 phase, and (e) S11 amplitude profile across the $\mathrm{C} 10$ and $\mathrm{C} 18$ SAM layers measured along the horizontal dashed line drawn in (c). Reprinted with permission from S. Wu, J.-J. Yu, Appl. Phys. Lett. 97, 202902 (2010), Copyright 2010, American Institute of Physics. 


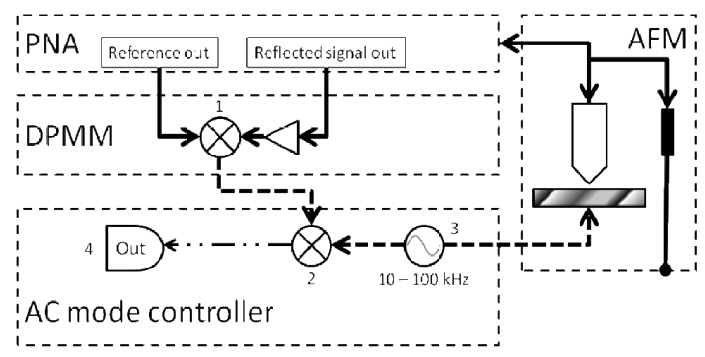

Fig. 5. Schematic setup of SMM for dopant density measurements: 1 - MW mixer for demodulation, $2-$ low frequency mixer for demodulating the modulated signal, $3-$ low frequency oscillator, $4-\mathrm{d} C / \mathrm{d} V$ data acquisition.

Nevertheless the different molecules in the two regions lead to different friction behavior of the AFM tip which is readily detected. Furthermore, the SAMs act as a thin dielectric between tip and gold substrate. The change in dielectric thickness results in a change of capacitance and is detected as a contrast in the reflected MW signal. The difference in the $S 11$ signal can be converted to a capacitance of $24 \mathrm{aF}$, Fig. 4 [22]. This value is consistent with a dielectric constant of 2 for the SAMs and an effective tip diameter of $60 \mathrm{~nm}$ which has been verified by electron micrograph investigation of the tip.

\subsection{Application to semiconductors}

The tip-sample impedance can also be affected by external parameters e.g. an electric field. The latter is well known behavior in semiconductors and especially in metal-insulator-semiconductor junctions (MIS). Many semiconductors like silicon or GaAs form an insulating oxide layer when exposed to oxygen or air. A metallic SMM tip touching a semiconductor surface in ambient conditions forms an MIS junction. When biasing the SMM tip at $V_{\mathrm{t}}$ the charge carriers in the semiconductor are depleted or attracted at the surface and a space charge region is formed. The thickness of the space charge region varies with $V_{\mathrm{t}}$ which affects the capacitance of the MIS junction [23]. The width of the space charge region is also a function of the charge carrier density in the semiconductor (which in many cases is equal to the concentration of impurity donor or acceptor atoms i.e. the dopant density).

Acquiring capacitance versus voltage curves $(C-V)$ would be too time consuming for mapping dopant densities over entire semiconductor topographies.

A bias modulation technique and subsequent signal recovery with lock-in amplifier techniques can be employed to measure the slope $\mathrm{d} C / \mathrm{d} V$ of the $C-V$ curve, Fig. 5 . The $\mathrm{d} C / \mathrm{d} V$ signal can then be calibrated on suitable calibration standards with known dopant density distributions.

Figure 6 shows a topography and dopant density map of a calibration standard sample [24]. A detailed description of the methods and results for dopant density

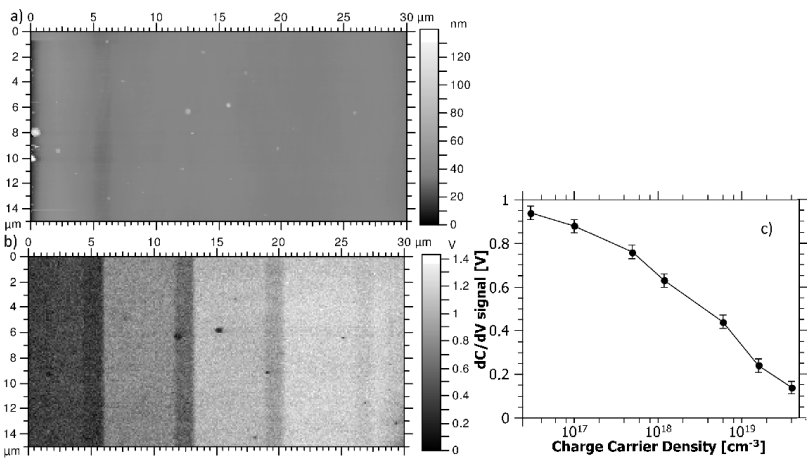

Fig. 6. SMM data of dopant density calibration standard sample. (a) AFM contact mode topography showing a few adsorbates, (b) the corresponding map of $\mathrm{d} C / \mathrm{d} V$ signal varying on the differently doped regions and (c) the corresponding transfer function of $\mathrm{d} C / \mathrm{d} V$ signal versus dopant density.

calibration in the range from $10^{14}$ atoms $/ \mathrm{cm}^{3}$ to $10^{20}$ atoms $/ \mathrm{cm}^{3}$ will be published elsewhere [25]. The dopant density measurement has empirically been shown to have a resolution of about $15 \mathrm{~nm}$ or better [26].

\section{Conclusion}

A scanning microwave microscope setup has been introduced which combines the high spatial resolution of an AFM with the impedance sensitivity of a modern vector network analyzer. Existing network analyzers are not sensitive for impedances that significantly deviate from $50 \Omega-$ as in the case of an AFM tip to sample contact. A resonator setup including the tip sample junction is implemented which effectively presents a $50 \Omega$ load to the PNA and brings it into the most sensitive range. The SMM has been used to map impedances in the attofarad range, dopant densities in the range from $10^{14}$ atoms $/ \mathrm{cm}^{3}$ to $10^{20}$ atoms $/ \mathrm{cm}^{3}$, and spatial resolutions of $15 \mathrm{~nm}$.

\section{References}

[1] G. Binnig, C.F. Quate, C. Gerber, Phys. Rev. Lett. 56, 930 (1986).

[2] S. Magonov, J. Alexander, S. Wu, in: Scanning Probe Microscopy of Functional Materials: Nanoscale Imaging and Spectroscopy, Eds. S.V. Kalinin, A. Gruverman, Springer, Berlin 2010, p. 233.

[3] R. Bennewitz, H.J. Hug, E. Meyer, Scanning Probe Microscopy: The Lab on a Tip, Springer, Berlin 2003.

[4] V.V. Talanov, A. Scherz, R.L. Moreland, A.R. Schwartz, Appl. Phys. Lett. 88, 134106 (2006).

[5] J. Kim, K. Lee, B. Friedman, D. Cha, Appl. Phys. Lett. 83, 1032 (2003).

[6] M. Tabib-Azar, D.-P. Su, A. Pohar, Rev. Sci. Instrum. 70, 3083 (1999).

[7] A. Imtiaz, S.M. Anlage, J.D. Barry, J. Melngailis, Appl. Phys. Lett. 90, 143106 (2007). 
[8] A. Karbassi, D. Ruf, A.D. Bettermann, C.A. Paulson, D.W. van der Weide, H. Tanbakuchi, R. Stancliff, Rev. Sci. Instrum. 79, 094706 (2008).

[9] W. Brezna, M. Schramboeck, A. Lugstein, S. Harasek, H. Enichlmair, E. Bertagnolli, E. Gornik, J. Smoliner, Appl. Phys. Lett. 83, 4253 (2003).

[10] A. Imtiaz, S.M. Anlage, Ultramicroscopy 94, 209 (2003).

[11] C. Eckhardt, W. Brezna, O. Bethge, E. Bertagnolli, J. Smoliner, J. Appl. Phys. 105, 113709 (2009).

[12] Scanning Probe Microscopy, Eds. S. Kalinin, A. Gruverman, Springer, New York 2007.

[13] B.T. Rosner, D.W. van der Weide, Rev. Sci. Instrum. 73, 2505 (2002).

[14] J. Smoliner, W. Brezna, Rev. Sci. Instrum. 78, 106104 (2007).

[15] G. Gomilla, J. Toset, L. Fumagalli, J. Appl. Phys. 104, 024315 (2008).

[16] Š. Lányi, Ultramicroscopy 103, 221 (2005).

[17] K. Lai, W. Kundhikanjana, H. Peng, Y. Cui, M.A. Kelly, Z.X. Shen, Rev. Sci. Instrum. 80, 043707 (2009).

[18] H.P. Huber, M. Moertelmaier, T.M. Wallis, C.J. Chiang, M. Hochleitner, A. Imtiaz, Y.J. Oh, K. Schilcher, M. Dieudonne, J. Smoliner, P. Hinterdorfer, S.J. Rosner, H. Tanbakuchi, P. Kabos, F. Kienberger, Rev. Sci. Instrum. 81, 113701 (2010).
[19] S. Xu, G.Y. Liu, Langmuir 13, 127 (1997).

[20] G.E. Poirier, E.D. Pylant, Science 272, 1145 (1996).

[21] M.D. Porter, T.B. Bright, D.L. Allara, C.E.D. Chidsey, J. Am. Chem. Soc. 109, 3559 (1987).

[22] S. Wu, J.-J. Yu, Appl. Phys. Lett. 97, 202902 (2010).

[23] Physics of Semiconductor Devices, Ed. S.M. Sze, Wiley, New York 1981.

[24] T. Clarysse, M. Caymax, P. De Wolf, T. Trenkler, W. Vandervorst, J.S. McMurray, J. Kim, C.C. Williams, J.G. Clark, G. Neubauer, J. Vac. Sci. Technol. B 16, 394 (1998).

[25] H.-P. Huber, I. Humer, M. Fenner, M. Moertelmaier, C. Rankl, A. Imtiaz, T.M. Wallis, H. Tanbakuchi, P. Hinterdorfer, P. Kabos, J. Smoliner, J. Kopansky, F. Kienberger, manuscript in preparation.

[26] W. Han, Agilent Application Note 5989-8881EN Rev A, Chandler 2008. 